\title{
Nurse-Led diabetes clinics in Southeast Asia: scope, feasibility, challenges and facilitators
}

\begin{abstract}
Burden of diabetes has escalated worldwide and global death rate has doubled in last two decades. About 88 million people are living with diabetes in Southeast Asian countries and it is expected to shoot up rapidly because of changing life style and longevity of life. Unfortunately, diabetes is not well managed in these countries because of several factors but mainly due to poor doctor-to-population ratio. Recently, World Health Organization has also advised accessible, affordable and quality diabetes care in Southeast Asian countries through extended role of nurses in diabetes care. The model of independent nurse-led diabetes clinic and advanced diabetes nurse practitioner for management of diabetes has been successful in the western world. This article highlights the scope, feasibility, challenges, barriers and facilitators in implementation of nurse-led diabetes clinics (NLDC) in Southeast Asian countries.
\end{abstract}

Keywords: nurse-led diabetic clinic, diabetes nurse, diabetes nurse practitioners, scope, feasibility, challenges, facilitators
Volume 7 Issue 2 - 2020

\author{
Suresh K Sharma,' Kalpana Thakur,' Ravi \\ Kant, ${ }^{2}$ Sanjay Kalra ${ }^{3}$ \\ 'College of Nursing, AllMS Rishikesh, India \\ 2Department of Medicine, AllMS Rishikesh, India \\ ${ }^{3}$ Department of Endocrinology, BRIDE Karnal, India
}

\section{Correspondence: Suresh K Sharma, PhD, RN, Professor \& Principal, College of Nursing, All India Institute of Medical Sciences, Rishikesh, Uttarakhand, India, Ph: 8475000293 ,} Email skaiims7@gmail.com

Received: April 14, 2020 | Published: June 09, 2020
Abbreviations: NLDC, nurse-led diabetes clinics; WHO, world health organization

\section{Introduction}

Globally, about 463 million people are living with diabetes, which is expected to be 700 million by 2045 . Out of which, 88 million adults are living with diabetes in Southeast Asian countries (India-72.0, Bangaldesh-8.4, Sri Lanka-1.2, Nepal-0.7 and Mauritius-0.2), which is estimated to increase to 153 million by 2045. ${ }^{1}$ The global death rate from diabetes has doubled in the last twenty years and World Health Organization (WHO) is predicting that there will be two third increase in this number by 2030 . $^{2}$ Southeast Asian countries have a poor doctor-to-population ratio which is one of the main factor that more than half of the people living with diabetes $(57 \%)$ are left undiagnosed ${ }^{1}$ and significant number of diabetes patients are left undertreated. ${ }^{4,5}$ There is high work load of diabetes patients for physicians in primary, secondary and tertiary care settings, which leads to poor quality of care and lack of proper follow-up. ${ }^{6}$ Studies reported very poor control of diabetes and significant high prevalence of diabetes related morbidities and mortalities. ${ }^{4,5}$ It is clearly evident that Southeast Asian countries especially India is having high burden of poor glycaemic control and early onset of complications in patients with diabetes. Recently, WHO advised to explore possibilities of lowcost alternative health care manpower like diabetes nurses for the effective management of diabetes in low-middle income countries. ${ }^{2}$ Nurses are the largest healthcare human resource available at all the level of health care delivery system including primary, secondary and tertiary health care settings. The model of nurseled clinic or advanced nurse practitioners has been adapted and well established in the western world for decades, which has been found empirically effective in the management of chronic illness including diabetes. Recently, randomized controlled trial conducted in University of Melbourne, Vitoria, Australia ${ }^{7}$ and University Putra Malaysia, Selangore, Malaysia ${ }^{8}$ found that nurse-led diabetes clinic care model was associated with increased anti-diabetic treatment initiation rates in primary care, and improvements in glycaemic control. This signifies that this model could also be implemented for the management of diabetes in Southeast Asian countries; however, there are few pertinent questions which require clear answers. Is it feasible to implement independent nurse-led diabetes care model in Southeast Asian countries? What would be scope of practice for diabetes nurses? What could be challenges and barriers in implement of this model? And what will be facilitators which can help mitigate barriers to implementation of this model? This comprehensive evidenced based review offers the answers to these pertinent questions

\section{Search strategy}

We summarized evidence regarding the scope, feasibility, challenges, barriers and facilitators in implementation of nurse-led diabetes clinics in Southeast Asian countries. We browsed PubMed, Embase and Scopus databases using the keywords with country (Southeast Asia) AND (India) restricted searches applied: (advanced diabetes nurse practitioners OR diabetes nurse) AND (Diabetes care); (nurse-led diabetes clinic) AND (diabetes care); (Diabetes care) AND (nurses); (nurses) AND (advanced nursing practice); (Diabetes) AND (nurses). We also screened the reference list of the selected articles to find other relevant articles.

\section{Can nurses manage diabetes effectively?}

There are sufficient evinces from the western world that nurses can manage diabetes effectively. Studies conducted in western world have highlighted significant effectiveness of nurse-led diabetes care model in improving glycaemic control, prevention of diabetes related complications and patient satisfaction with their treatment. ${ }^{9-11}$ In one of qualitative study, a diabetes patient verbalised that "nurses are my saviour, I was felt ignored previously by conventional diabetes care model". ${ }^{10}$ The insulin titration done by nurses through nurse-led follow-up clinic along with diabetes educational measures was found to be equally effective as physician driven diabetes management in lowering patients' HbAlc levels in some of the studied. ${ }^{9,10}$ Randomized controlled studies on efficacy of nurse-led diabetes clinic in management of diabetes highlighted 
that insulin dosage titration, collaboration with other team members along with patients' involvement in treatment result in dramatic improvement of blood glucose level among poorly controlled diabetic patients. ${ }^{10-13}$ Monthly diabetic clinic run by nurses proven to be effective among African diabetic people and study results show that there was a significant improvement in $\mathrm{HbA} 1 \mathrm{c}$ level from baseline to 18 months. ${ }^{12}$ Thus, it is clearly evident that nurses with specialized training and experience can effectively manage diabetes

\section{Scope of practice for diabetes nurse practitioners}

American Nursing Association ${ }^{14}$ mentions that advanced nurse practitioners, and clinical nurse specialists play a pivotal role in the future of health care; they can assess, diagnose, manage illnesses, advise the public on health issues, manage chronic diseases, and engage in continuous education to remain ahead of any technological, methodological, or other developments in the field. It is evident from studies that diabetes nurses working in nurse-led diabetes clinics can efficiently carryout detailed physiological assessment, prescribe blood tests, plan for treatment modification, manage dose titration of insulin or other anti-diabetes drugs, monitor patients for early recognition of complications in long term, perform health education, provide follow-up services and do referral to medical specialists whenever indicated. ${ }^{15-19}$ A summary on the scope of practice for advanced diabetes nurse practitioners has been presented in Table 1. Nurse-led diabetes clinic as an adjunct to the hospital based diabetes care is a cost-effective alternative for the effective management of diabetes and its related morbidities such as hypertension and hyperlipidaemia through focused assessment, education, behavioural modification, motivation for the lifestyle changes, follow-up for monitoring and treatment adherence for glycaemic control and prevention of diabetes related complications. ${ }^{20}$ NLDC can offer wide variety of services like assessment, ordering laboratory test, prescribing and titrating insulin and other anti-diabetic drugs, monitoring glycaemic control and compliance with treatment and life-style modification advices, diabetes education for patients and family regarding self-care efficacy building, dietary advices, lifestyle modification (exercise, yoga, medication, stress reduction activities etc.) motivation, collaborating between patient and physician along with follow-up and referral services. ${ }^{21}$ Advanced diabetes nurse practitioners can work at primary, secondary and tertiary heath care setting in adjunct or independent role. ${ }^{15-19}$ An evidence based model is proposed for scope of nurse-led diabetes clinic, which is illustrated in Figure 1.

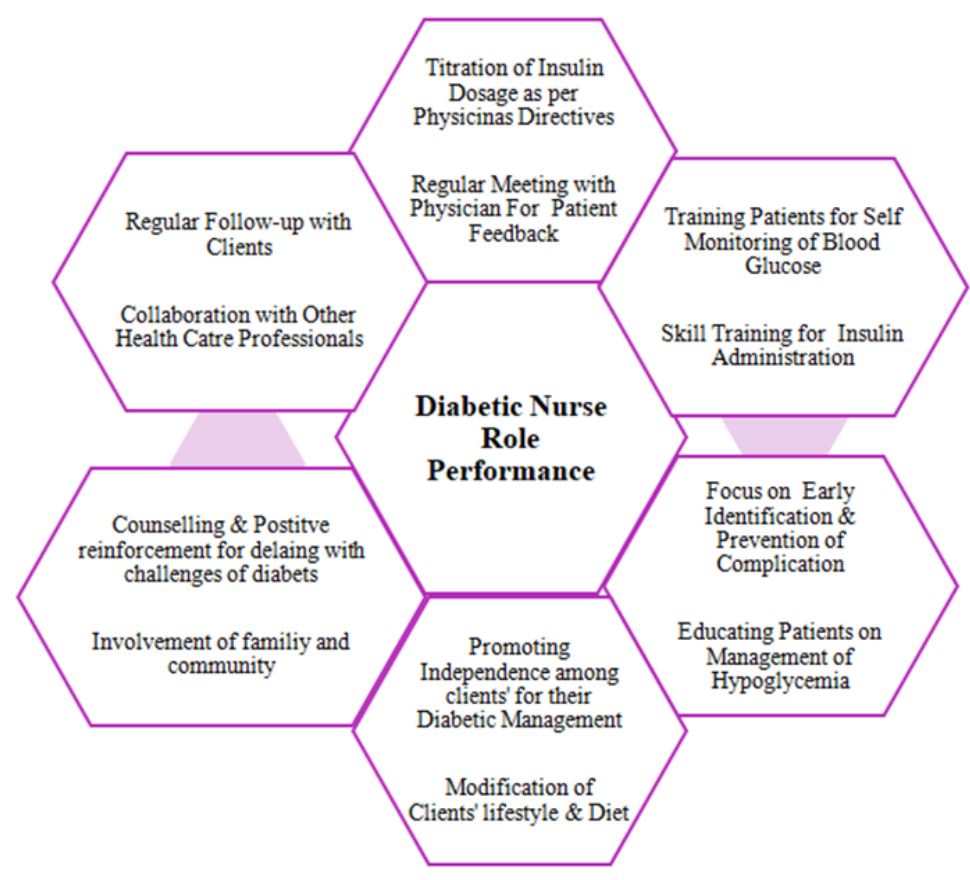

Figure I Model of adjunct nurse-led diabetes clinic: scope of practice.

Table I Scope of practice for the advanced diabetes nurse practitioners ${ }^{[15-19]}$

\section{I.Assessment}

2. Diagnosis of diabetes and its complications

3. Prescribing and dose titration of insulin and other anti-diabetic drugs

4. Ordering tests like blood glucose, HbAIc, KFT, LFT and haemogram etc.

5. Health promotion and diabetes prevention activities

6. Patient and family education to promote self-care efficacy

7. Monitoring for compliance with prescribed treatment and life style modification advices.
8. Collaborative resource for diabetes specialist

9. Follow-up services

10. Referral services to medical specialist when indicated.

II. Serving rural, underserved and vulnerable population

12. Mentoring, teaching and research

13. Patient advocacy

14. Administrative and managerial activity

Advanced diabetes nurse practitioners can work at primary, secondary and tertiary heath care setting in adjunct or independent role.

KFT, kidney function test; LFT, liver function test 


\section{Feasibility of nurse-led diabetes clinics}

In Southeast Asian countries, diabetes care is primarily provided by the general physicians ${ }^{4}$ because specialists are in very short supply in these countries. The burden of diabetes is rapidly growing, thus existing general physicians and diabetes specialists are overloaded, which reflect a significant inadequacy in the quality of diabetes care. A recent study reported, average daily load of diabetes patients on a specialities is 400 and it is about 100 patients for each doctor in primary health care setting; because of which quality and follow-up of diabetes care suffered seriously. ${ }^{4}$ It is clearly evident that existing medical human resources for management of diabetes in Southeast Asian countries, especially in India are falling seriously short to the tsunami of diabetes, consequently people are left undiagnosed, inadequately treated, and poorly followed-upand increased diabetes related morbidities and mortality.Therefore, there is serious need of nurse-led diabetes clinics through exiting primary health care model..$^{22}$ National Health Policy, 2017 of India also highlights need of creating middle level healthcare service providers and creating cadres for nurse practitioners and starting specialized nursing training courses. ${ }^{23}$ There is sufficient literature that supports the effectiveness and acceptability of nurse-led clinics in western world. ${ }^{9-11}$ Most of these studies found nurse-led clinics were equivocal in quality of care and patient satisfaction; if not superior when compared with existing conventional model of care for chronic diseases including diabetes. The nurse-led diabetes clinic when used as an adjunct to existing diabetes care services; it was found to be a cost-effective and efficacious alternative for effective management diabetes..$^{20}$ Studies from India has reported feasibility to start the nurse-led clinic for post-myocardial infract patients ${ }^{17}$ and diabetes patients with adequate specialized training, legislative authority and creation of such specialized cadre for nurses. ${ }^{24}$ Initially, it could be started as integrated-adjunct model with physician's clinics for diabetes care and later they may independently handle the cases; as this model was recently found successful in Singapore. ${ }^{13}$ This multidisciplinary model of diabetes care will not only reduce the work-load of physicians, but also improve the affordability, accessibility and quality of diabetes care in these low resource countries.

\section{Challenges, barriers and facilitators}

A large cohort study from India reported very poor control of diabetes (mean $\mathrm{HbA} 1 \mathrm{c}=9.2 \%$ ) and high prevalence of diabetes related complications, which was primarily because of suboptimal insulin prescription, poor compliance and lack of proper followup. ${ }^{6}$ There are several challenges in Southeast Asian countries for the effective management of diabetes, some of most crucial are large population, low doctor-to-population ratio, illiteracy, weak primary health care services, low budget for health care, poverty, clinical inertia in doctors to prescribe insulin, lack of adherence with prescribed treatment etc. However, poor controlled diabetes may have significant negative impact on health and economy of the community and country. Therefore, the nurse-led diabetes clinic model will be a best alternative solution for these resourceconstraint countries, which has already been proven to be efficacious and cost-effective in western world and other countries including Africa. However, implementation of nurse-led diabetic clinics is not an easy task in Southeast Asian region because of restrictive government legislations, regulations, inadequate training of nurses, poor infrastructure, lack of acceptance by medical colleagues and patients, lack of awareness and confidence on nurse-led care and most significant constraints are dominance of medical fraternity, perceived lower status of nursing profession and weak national nursing leadership. Table 2 Nurses' potentials are unrecognized and they are underutilised in Southeast Asian countries as it is observed that most of the nurses with specialized qualification are either working as a general nurse or nurse educators. Therefore, Le Baron et al., ${ }^{25}$ recommends to optimize role of the nurse and explore innovative models of care delivery, empower staff nurses, strengthen nurse leadership, offer relevant educational programs, enhance teamwork for promoting their extended role as advanced nurse practitioner in India. ${ }^{25}$ Furthermore, a list of facilitators to overcome the challenges and barriers in implementation of advanced diabetes nurse practitioners has been illustrated in Table 3 .

Table 2 Challenges, and barriers to implement nurse-led diabetes clinics ${ }^{[15-19]}$

Organisation and policy related factors

- Restrictive government legislation and regulations

(no prescribing, tests ordering, dose titrating and referral rights for nurses)

- Uncertainty about medico-legal obligations and responsibilities of nurses

- Lack of support from health leaders and government

- Lack of infrastructure and support

- Weak national nursing leadership

Individual factors

- Lack of confidence in competence of ability to carry out advanced diabetes care role

- Lack of prescribing and autonomous decision making ability

- Doctors lack confidence in quality of training, knowledge and skills of nurses to take advanced diabetes care role

- Lack of acceptance by patients and negative belief about nurses and preference to consult medical professionals

Training and education

- Lack of confidence about adequacy of training and education of nurses to take advanced diabetes care role

- Lack of trained faculty and educational infrastructure

- Lack of support from medical colleagues in teaching and training

Team factors

- Lack of awareness about advanced role of nurses in public and doctor colleagues

- Lack of acceptance of advanced role of nurses by doctors and other health care colleagues

- Difficulties in collaborative relationship with inter-professional (e.g. consultants, resident doctors) and intra-professional (other nurses) groups

- Lack of clear understanding about scope of advanced diabetes nurses practice

- Varied expectations about advanced role of nurses in diabetes care

- Financial competition between doctors and nurses in private practice

Societal factors

- Perceived poor image of nursing profession in society in Southeast Asian countries

- Lack of awareness and confidence in nurses' capability to provide diabetes care independently

- Medical fraternity dominated society

- Nursing is not perceived as independent discipline in SEA countries 
Table 3 Facilitators for implementation of nurse-led diabetes clinics ${ }^{[15,25]}$

Organisation and policy related factors

- Government legislation and regulations must be facilitative for prescribing, ordering tests, insulin and anti-diabetic drugs, dose titration and referral rights to the advanced diabetes nurse practitioners (ADNPs)

- Clear policy about medico-legal responsibilities of ADNPs

- Support from health leaders and government with sufficient funds

- Provision of adequate infrastructure and support by organization

- Leadership capacity building for national nursing leaders

Individual factors

- Adequate training, competence, motivation of nurses to carry out advanced diabetes care roles

- Implementation of nursing practice act in Southeast Asian countries with precisely prescribed autonomy, and prescribing rights for the diabetes nurse practitioners.

- Collaborative model of training, education and adjunct model of nurse-led diabetes clinic help achieve doctors' confidence in capabilities of diabetes nurses

- Nursing societies and association must work for promoting public awareness about advanced diabetes nurse practitioners' role and publicize their success stories

Training and education

- Sound curriculum, training model, skill development activities and evaluation system to produce knowledgeable and skilled advanced diabetes nurse practitioners

- Sound training of faculty to promote excellent mentoring and education for diabetes nurses.

- Competency based collaborative training by qualified medical and nursing faculty to produce skilled ADNPs

Team factors

- Building supportive relationship between doctors, other health care professionals and ADNPs

- Nursing regulatory bodies must implement nursing practice acts and clearly define the scope of advanced diabetes nurses practice to avoid any ambiguity

- Clearly defined and distinguished independent, interdependent and collaborative roles of diabetes nurses will avoid any confusion, resistance and conflict with their medical colleagues

Societal factors

- Promoting awareness about advanced diabetes nurse practitioners' role

- Brand-building activities for advanced independent role of nurses in health care delivery system of SEA region

- National societies and associations of diabetes nurses must propagate the success stories of diabetes nurses to gain confidence of public in their services

- Nurses must be recognized as partners of care, active involvement in policy making, decision making, autonomy in care delivery and administration

- Clearly defined roles, better pay and autonomy will automatically attract best talent pool of society and recognition as important partners in care delivery system of the country

\section{Conclusion}

There is growing recognition for need of high quality diabetes care in Southeast Asian countries, because of continuous report about poor diabetes control and consequently high diabetes related morbidities and mortalities. Nurse-led diabetes clinic model of diabetes care is one of efficacious and cost-effective alternative to achieve accessible, affordable and quality diabetes care in these resource-constraint countries. There are challenges, barriers in implementation of this model of diabetes care such as restrictive government legislations, regulations, inadequate training of nurses, poor infrastructure, lack of acceptance by medical fraternity and patients, lack of awareness \& confidence on nurse-led care, dominance of medical fraternity, perceived lower status of nursing profession and weak national nursing leadership. However, this model for diabetes care has been successfully working in developed countries since decades; which is practically very much possible in southeast Asian counties with strong government will, adequate training, legislative and regulatory authority to nurses.

\section{Acknowledgments}

None.

\section{Conflicts of interest}

The authors declare that there are no conflicts of interest.

\section{Funding}

None.

\section{References}

1. International Diabetes Federation. IDF Diabetes Atlas 9th edition. 
2. World Health Organisation. Addressing Asia's fast growing diabetes epidemic. Bulletin of the World Health Organization. 2017;95(8):550 551.

3. Chandan H. As India struggles with doctor shortage, govt. gives a push to nurse-led clinics. 2018

4. Tripathy JP, Sagili KD, Kathirvel S, et al. Diabetes care in public health facilities in India: a situational analysis using a mixed methods approach. Diabetes Metab Syndr Obes. 2019;12:1189-1199.

5. Raheja BS, Kapur A, Bhoraskar A, et al. DiabCare Asia-India study: diabetes care in India-current status. $J$ Assoc Physicians India. 2001;49:717-722.

6. Mohan V, Shah S, Saboo B. Current glycemic status and diabetes related complications among type 2 diabetes patients in India: data from the A1chieve study. J Assoc Physicians India. 2013;61(1 Suppl):12-15.

7. Furler J, O'Neal D, Speight J, et al. Supporting insulin initiation in type 2 diabetes in primary care: results of the stepping up pragmatic cluster randomised controlled clinical trial. BMJ. 2017;356:j783.

8. Azami G, Soh K1, Sazlina SG, et al. Effect of a nurse-led diabetes selfmanagement education program on glycosylated hemoglobin among adults with type 2 diabetes. J Diabetes Res. 2018:4930157.

9. Swinnen SG, Hoekstra JB, DeVries JH. Insulin therapy for type diabetes. Diabetes Care. 2009;32(Suppl 2):S253-S259.

10. Thompson DM, Kozak SE, Sheps S. Insulin adjustment by a diabetes nurse educator improves glucose control in insulin-requiring diabetic patients: a randomized trial. CMAJ. 1999;161(8):959-962.

11. Yki-Jarvinen $\mathrm{H}$, Juurinen $\mathrm{L}$, Alvarsson $\mathrm{M}$, et al. Initiate insulin by aggressive titration and education (INITIATE): a randomized study to compare initiation of insulin combination therapy in type 2 diabetic patients individually and in groups. Diabetes Care. 2007;30(6):1364 1369.

12. Price C, Shandu D, Dedicoat M, et al. Long-term glycaemic outcome of structured nurse-led diabetes care in rural Africa. QJM. 2011;104(7):571574
13. Dehghan Nayeri N, Samadi N, Larijani B, et al. Effect of nurse-led care on quality of care and level of $\mathrm{HbA1C}$ in patients with diabetic foot ulcer: A randomized clinical trial. Wound repair regen. 2020;28(3):338-346.

14. American Nurses Association. Advanced Practice Registered Nurse (APRN).

15. Torrens C, Campbell P, Hoskins G, et al. Barriers and facilitators to the implementation of the advanced nurse practitioner role in primary care settings: a scoping review. Int J Nurs Stud. 2020;104:103443.

16. Usha P, Gopichandran L, Pathak P, et al. A study to evaluate the effect of a nurse-led follow-up clinic among postmyocardial infraction patients attending the cardiac outpatient department at CN centre, AIIMS, New Delhi. J Pract Cardiovasc Sci. 2018;4(3):198-205.

17. Hatchett R. Key issues in setting up and running a nurse-led cardiology clinic. Nurs Stand. 2005;20(14-16):49-53.

18. Harbman P. The development and testing of a nurse practitioner secondary prevention intervention for patients after acute myocardial infarction: a prospective cohort study. Int J Nurs Stud. 2014;51(12):1542-1556.

19. Al-Mallah MH, Farah I, Al-Madani W, et al. The impact of nurse-led clinics on the mortality and morbidity of patients with cardiovascular diseases. J Cardiovasc Nurs. 2016;31(1):89-95.

20. Mason JM, Freemantle N, Gibson M, et al. Specialist nurse-led clinics to improve control of hypertension and hyperlipidemia in diabetes: economic analysis of the SPLINT trial. Diabetes Care. 2005;28(1):40-46.

21. Carey N, Courtenay M. A review of the activity and effects of nurse-led care in diabetes. J Clinic Nurs. 2007;16(11C):296-304.

22. Basu S, Sharma N. Diabetes self-care in primary health facilities in Indiachallenges and the way forward. World J Diabetes. 2019;10(6):341-349.

23. Ministry of Health and Family welfare. National Health Policy. 2017.

24. Joshi M, Ukande U. Feasibility of nurse-led diabetes clinic: a pilot study. IOSR Journal of Applied Geology and Geophysics. 2016;4(6):1-17.

25. Le Braron VT, Palat G, Sinha S, et al. Recommendations to support nurses and improve the delivery of oncology and palliative care in India. Indian J Palliat Care. 2017;23(2):188-198. 